\title{
Molecular Hydrogen Formation on Astrophysically Relevant Surfaces
}

\author{
N. Katz, ${ }^{1}$ I. Furman, ${ }^{1}$ O. Biham, ${ }^{1}$ V. Pirronello, ${ }^{2}$ and G. Vidali ${ }^{3}$ \\ ${ }^{1}$ Racah Institute of Physics, The Hebrew University of Jerusalem, Jerusalem 91904, Israel \\ ${ }^{2}$ Istituto di Fisica, Universita' di Catania, 95125 Catania, Sicily, Italy \\ ${ }^{3}$ Department of Physics, Syracuse University, Syracuse, NY 13244
}

Received — 


\begin{abstract}
Recent experimental results about the formation of molecular hydrogen on astrophysically relevant surfaces under conditions close to those encountered in the interstellar medium are analyzed using rate equations. The parameters of the rate equation model are fitted to temperature-programmed desorption curves obtained in the laboratory. These parameters are the activation energy barriers for atomic hydrogen diffusion and desorption, the barrier for molecular hydrogen desorption, and the probability of spontaneous desorption of a hydrogen molecule upon recombination. The model is a generalization of the Polanyi-Wigner equation and provides a description of both first and second order kinetic processes within a single model. Using the values of the parameters that fit best the experimental results, the efficiency of hydrogen recombination on olivine and amorphous carbon surfaces is obtained for a range of hydrogen flux and surface temperature pertinent to a wide range of interstellar conditions.
\end{abstract}

Subject headings: dust - ISM; abundances — ISM; molecules — molecular processes 


\section{Introduction}

The formation of molecular hydrogen in the interstellar medium (ISM) is a process of fundamental importance (Duley \& Williams 1984, Williams 1998). It was recognized long ago (Gould \& Salpeter 1963) that $\mathrm{H}_{2}$ cannot form in the gas phase efficiently enough to account for its abundance. It was proposed that dust grains act as catalysts allowing the protomolecule to quickly release the $4.5 \mathrm{eV}$ of excess energy in a time comparable to the vibration period of the highly vibrationally excited state in which it is formed. The problem can be described as follows. An $\mathrm{H}$ atom approaching the surface of a grain has a probability $\xi$ to become adsorbed. The adsorbed $\mathrm{H}$ atom (adatom) spends an average time $t_{\mathrm{H}}$ (residence time) before leaving the surface. If during the residence time the $\mathrm{H}$ adatom encounters another $\mathrm{H}$ adatom, an $\mathrm{H}_{2}$ molecule will form with a certain probability.

This problem has been studied theoretically over the years and different models have been proposed (Gould \& Salpeter 1963; Williams 1968; Hollenbach \& Salpeter 1970; Hollenbach \& Salpeter 1971; Hollenbach, Werner and Salpeter 1971; Smoluchowski 1981; Aronowitz \& Chang 1985; Duley \& Williams 1986; Pirronello \& Averna 1988; Sandford \& Allamandolla 1993; Takahashi et al. 1999; Farebrother et al. 1999). In particular, Hollenbach et al. calculated sticking and accommodation of $\mathrm{H}$ atoms in a semiclassical way, while the mobility was treated quantum mechanically. They concluded that tunneling between adsorption sites, even at $10 \mathrm{~K}$, would have assured the required mobility. The steady state production rate of molecular hydrogen per unit volume was expressed according to (Hollenbach, Werner and Salpeter 1971)

$$
R_{\mathrm{H}_{2}}=\frac{1}{2} n_{\mathrm{H}} v_{\mathrm{H}} \sigma \gamma n_{\mathrm{g}}
$$

where $n_{\mathrm{H}}$ and $v_{\mathrm{H}}$ are the number density and the speed of $\mathrm{H}$ atoms in the gas phase, respectively, $\sigma$ is the average cross-sectional area of a grain and $n_{\mathrm{g}}$ is the number density of dust grains. The parameter $\gamma$ is the fraction of $\mathrm{H}$ atoms striking the grain that eventually form a molecule, namely $\gamma=\xi \eta$, where $\eta$ is the probability that an $\mathrm{H}$ adatom on the surface will recombine with another $\mathrm{H}$ atom to form $\mathrm{H}_{2}$. The probability $\xi$ for an $\mathrm{H}$ atom to become adsorbed on a grain surface covered by an ice mantle has been calculated by Buch and Zhang (1991) and Masuda et al. (1998). Eq. (1) states that, for $\eta=1$, whenever two $\mathrm{H}$ atoms are adsorbed on a grain, a $\mathrm{H}_{2}$ molecule is formed.

Recently, a series of experiments were conducted to measure hydrogen recombination in an ultra high-vacuum (UHV) chamber by irradiating the sample with two beams of $\mathrm{H}$ and D atoms and monitoring the HD production rate (Pirronello et al. 1997a, Pirronello et al. 1997b, Pirronello et al. 1999). The two beams were used in order to obtain a better 
signal to noise ratio than would have been possible for $\mathrm{H}_{2}$. Two different substrates have been used: a natural olivine (a silicate made of a mixture of $\mathrm{Mg}_{2} \mathrm{SiO}_{4}$ and $\mathrm{Fe}_{2} \mathrm{SiO}_{4}$ ) slab; and an amorphous carbon sample. These samples are considered as better analogues of interstellar dust than any other model surface which was studied before. The substrate temperatures were in the range between $5 \mathrm{~K}$ and $15 \mathrm{~K}$. The HD formation rate was measured using a quadrupole mass spectrometer both during and after irradiation with $\mathrm{H}$ and D atoms. In the latter case, a Temperature Programmed Desorption (TPD) experiment was carried out in which the temperature of the sample was quickly ramped to over $30 \mathrm{~K}$ to desorb all weakly adsorbed species.

The main results obtained by Pirronello et al. (1997a, 1997b, 1999) are as follows: (a) In the temperature range of interest for interstellar applications (between $10 \mathrm{~K}$ and $15 \mathrm{~K}$ ), the formation rates deduced from the experimental data are about one order of magnitude lower than those calculated by and Hollenbach \& Salpeter $(1970,1971)$ and Hollenbach et al. (1971); (b) According to the desorption spectra, hydrogen, that is adsorbed as atomic, appears to acquire significant mobility only around $9 \mathrm{~K}$ in the case of olivine and at a somewhat higher temperature in the case of amorphous carbon, even in the high coverage regime. Thus, at temperatures lower than about 10K tunneling alone does not provide enough mobility to $\mathrm{H}$ adatoms to enable recombination, and thermal activation is required.

In this paper we perform a detailed analysis of the hydrogen recombination experiments of Pirronello et al. (1997a, 1997b, 1999) using a rate equation model. The parameters of the rate equation model are fitted to the experimental TPD curves. These parameters are the activation energy barriers for atomic hydrogen diffusion and desorption, the barrier for molecular hydrogen desorption and the probability of spontaneous desorption of a hydrogen molecule upon recombination. Using the values of the parameters that fit best the experimental results, the efficiency of hydrogen recombination on the olivine and amorphous carbon surfaces is obtained for a range of hydrogen fluxes and surface temperatures pertinent to a wide range of interstellar conditions.

The paper is organized as follows. In Sec. 2 we describe the experiments to be analyzed. The rate equation model is introduced in Sec. 3. Subsequent analysis and results are presented in Sec. 田, followed by a discussion in Sec. 5 and a summary in Sec. 6 .

\section{Review of Experimental Methods}

The experimental apparatus and measurement techniques are described in Pirronello et al. (1997a, 1997b, 1999) and in greater detail in Vidali et al. (1998a). Here we give a 
brief outline. The apparatus consists of an ultra-high vacuum (UHV) chamber pumped by a cryopump and a turbomolecular pump (operating pressure in the low $10^{-10}$ torr range). The sample is placed in the center of the UHV chamber and mounted on a liquid helium continuous flow cryostat. By varying the flow of liquid helium and with the use of a heater located behind the sample, temperatures can be maintained in the range of 5-30 K. For cleaning purposes, the temperature of the sample can be raised to about $200^{\circ} \mathrm{C}$ (without liquid helium in the cryostat). The temperature is measured by an iron-gold/chromel thermocouple and a calibrated silicon diode placed in contact with the sample. Two triple differentially pumped atomic beam lines are aimed at the surface of the sample. Each has a radio-frequency cavity in which the molecular species is dissociated, cooled to $\sim 200 \mathrm{~K}$ by passing the atoms through a cooled $\mathrm{Al}$ channel, and then injected into the line. Dissociation rates are typically in the 75 to $90 \%$ range, and are constant throughout a run. Estimated fluxes are as low as $10^{12}$ atoms $\mathrm{cm}^{-2} \mathrm{~s}^{-1}$ (Vidali et al. 1998a).

The reason for using two different lines and two isotopes (one line for $\mathrm{H}$ and the other for $\mathrm{D}$ ) is that in preliminary runs using only one line, it became evident that the signal of $\mathrm{H}_{2}$ formation was hidden in the background given by the undissociated fraction of molecules coming directly from the beam source. The possibility of using a second line is undoubtedly one of the most important features of this equipment. By using $\mathrm{H}$ atoms in one line and D atoms in the other, we can look at the formation of HD on the surface, knowing that there are no other spurious sources of HD. The signal of HD is collected by a quadrupole mass spectrometer mounted on a rotatable flange. The experiment is done in two phases. First, $\mathrm{H}$ and $\mathrm{D}$ beams are sent onto the surface for a given period of time (from tens of seconds to tens of minutes). At this time any HD formed and released is detected. In the second phase (the TPD phase), the sample temperature is quickly $(\sim 0.6 \mathrm{~K} / \mathrm{sec})$ ramped and the HD signal is measured.

By measuring the desorption rate $R(t)$ as a function of time, as well as the temperature of the surface as a function of time, information on the kinetics of desorption can be obtained. The desorption rate can by approximated by the Polanyi-Wigner equation:

$$
R(t)=\nu N(t)^{\beta} \exp \left(-E_{d} / k_{B} T\right)
$$

where $N$ is the number density of reactants on the surface, $\beta$ is the order of desorption, $\nu$ is the attempt frequency, $E_{d}$ is the effective activation energy for the dominant recombination and desorption process and $T=T(t)$ is the sample temperature. In the TPD experiment, first order $(\beta=1)$ desorption curves $R(t)$ exhibit asymmetric peaks with a sharp drop-off on the right hand side. The position of the peak is insensitive to coverage. Second order desorption curves $(\beta=2)$ exhibit symmetric peak shapes. These peaks shift towards lower 
temperatures as coverage is increased (Chan et al. 1978).

In the experiments analyzed here the irradiation stage was done at a surface temperature between $T=5 K$ and $T=7 K$, for several irradiation time intervals. The HD desorption rate vs. surface temperature during the TPD runs is shown in Figs. 1 and 2 for olivine and in Figs. 3 and 4 for amorphous carbon. The TPD curves shown in Figs. 1 and 3 exhibit first order kinetics due to the larger irradiation times, while the ones shown in Figs. 2 and 4 exhibit second order kinetics. For both the olivine and the amorphous carbon samples, at the sample temperature examined here, most of the HD detected is formed because of thermal activation during the heat pulse. Only a small fraction of HD is formed during the irradiation process, showing that, at least under our experimental conditions, prompt-reaction mechanisms (Duley \& Williams 1986) or fast tunneling (Hollenbach, Werner and Salpeter 1971) are not that important.

\section{Model}

\subsection{Assumptions}

In the desorption curves studied here most of the adsorbed hydrogen is released well before a temperature of $30 \mathrm{~K}$ is reached. Therefore, we assume that the hydrogen atoms on the surface are trapped in physisorption potential wells and are thus only weakly adsorbed. We also assume that the mechanism for the creation of $\mathrm{H}_{2}$ (or HD) is the Langmuir-Hinshelwood (LH) scheme, namely that the rate of creation of $\mathrm{H}_{2}$ is diffusion limited. This assumption is justified due to the observed Langmuir-like kinetics of the amounts desorbed as a function of the irradiation time (Pirronello et al. 1997a). Since the coverages involved in the experiments analyzed here are low, other mechanisms such as the Eley-Rideal (ER) scheme, in which hydrogen atoms, coming from the gas phase, collide and promptly react with already adsorbed hydrogen atoms, are of less importance and are not included in our model. Furthermore, the experimental results indicate that the ER mechanism does not contribute significantly, since even at higher coverages there was little desorption of HD during the irradiation phase.

The model we present here, which reproduces quite well the experimental desorption curves, and the choice of the assumptions on which it is based are the results of other less successful attempts. We have, in fact, tried to fit the desorption curves using a model in which the $\mathrm{H}$ and $\mathrm{D}$ populations of adatoms that are used in the experiments were described separately. In these earlier attempts we assumed that all HD molecules are promptly 
released upon formation, as investigations on more regular metal surfaces suggest (Rettner \& Auerbach 1996; Winkler 1998). The model in that case has four parameters: the diffusion barriers as well as the desorption barriers for $\mathrm{H}$ and $\mathrm{D}$ adatoms. We found that such model cannot provide a reasonable fit to the experimental desorption curves.

In the model we present here, we do not assume spontaneous desorption once a molecule is created on the surface despite the $\sim 4.5 \mathrm{eV}$ released in the recombination. In order not to increase the number of parameters to be used in the fits, we decided not to treat separately the two populations of $\mathrm{H}$ and $\mathrm{D}$ adatoms, but to consider only one population of $\mathrm{H}$ adatoms characterized by average properties. In this way we kept the number of parameters to four: the activation energy barriers for diffusion and desorption of hydrogen adatoms, the activation energy barrier for desorption of molecular hydrogen that had not been released into the gas phase upon formation, and the probability $1-\mu$ of spontaneous desorption of a hydrogen molecule upon recombination.

An important final assumption is that all energy barriers are coverage independent. This assumption may not apply at high coverage. However, at the low coverages obtained in the experiments analyzed here (up to $\sim 1 \%$ of a layer), it is a reasonable assumption.

\subsection{Rate Equations}

Consider an experiment in which a flux of $\mathrm{H}$ atoms is irradiated on the surface. $\mathrm{H}$ atoms that stick to the surface, once the surface temperature is raised, perform hops as random walkers with increased frequency and recombine when they encounter one another. Let $N_{1}(t)$ [in monolayers $(M L)$ ] be the coverage of $\mathrm{H}$ atoms on the surface and $N_{2}(t)$ (also in $M L)$ the coverage of $\mathrm{H}_{2}$ molecules. We obtain the following set of rate equations:

$$
\begin{aligned}
& \dot{N}_{1}=F\left(1-N_{1}-N_{2}\right)-P_{1} N_{1}-2 \alpha N_{1}^{2} \\
& \dot{N}_{2}=\mu \alpha N_{1}^{2}-P_{2} N_{2} .
\end{aligned}
$$

The first term on the right hand side of Eq. (3a) represents the incoming flux in the Langmuir kinetics. In this scheme $\mathrm{H}$ atoms deposited on top of $\mathrm{H}$ atoms or $\mathrm{H}_{2}$ molecules already on the surface are rejected. F represents an effective flux (in units of $M L s^{-1}$ ), namely it already includes the possibility of a temperature dependent sticking coefficient. The second term in Eq. (3a) represents the desorption of $\mathrm{H}$ atoms from the surface. The desorption coefficient is 


$$
P_{1}=\nu \cdot \exp \left(-E_{1} / k_{B} T\right)
$$

where $\nu$ is the attempt rate (standardly taken to be $10^{12} \mathrm{~s}^{-1}$ ), $E_{1}$ is the activation energy barrier for desorption of an $\mathrm{H}$ atom and $T$ is the temperature. The third term in Eq. (3a) accounts for the depletion of the $\mathrm{H}$ population on the surface due to recombination into $\mathrm{H}_{2}$ molecules, where

$$
\alpha=\nu \cdot \exp \left(-E_{0} / k_{B} T\right)
$$

is the hopping rate of $\mathrm{H}$ atoms on the surface and $E_{0}$ is the activation energy barrier for $\mathrm{H}$ diffusion. Here we assume that there is no barrier for recombination. If such a barrier is considered, it can be introduced as discussed in Pirronello et al. (1997b, 1999). The first term on the right hand side of Eq. (3b) represents the creation of $\mathrm{H}_{2}$ molecules. The factor 2 in the third term of Eq. (3a) does not appear here since it takes two $\mathrm{H}$ atoms to form one molecule. The parameter $\mu$ represents the fraction of $\mathrm{H}_{2}$ molecules that remains on the surface upon formation, while a fraction of $(1-\mu)$ is spontaneously desorbed due to the excess energy released in the recombination process. The second term in Eq. (3b) describes the desorption of $\mathrm{H}_{2}$ molecules. The desorption coefficient is

$$
P_{2}=\nu \cdot \exp \left(-E_{2} / k_{B} T\right),
$$

where $E_{2}$ is the activation energy barrier for $\mathrm{H}_{2}$ desorption. The $\mathrm{H}_{2}$ production rate $R$ is given by:

$$
R=(1-\mu) \cdot \alpha N_{1}^{2}+P_{2} N_{2} .
$$

This model can be considered as a generalization of the Polanyi-Wigner model [see Eq. (2)]. It gives rise to a wider range of simultaneous applications, compared to Eq. (2). In particular, it describes both first order and second order desorption kinetics (or a combination) for different regimes of temperature and flux.

In the experiments analyzed here, both the temperature and the flux were controlled and monitored throughout. Each experiment consists of two phases. In the first phase the sample temperature is constant up to time $t_{0}$, under a constant irradiation rate $F_{0}$. In the second phase, the irradiation is turned off and linear heating of the sample is applied at the rate $b\left(K s^{-1}\right)$ : 


$$
\begin{array}{lll}
F(t)=F_{0} ; & T(t)=T_{i}: & 0 \leq t<t_{0} \\
F(t)=0 ; & T(t)=T_{i}+b\left(t-t_{0}\right): & t \geq t_{0} .
\end{array}
$$

Here $T_{i}$ is the constant temperature of the sample during irradiation.

In the case that the rejection terms in $F\left(1-N_{1}-N_{2}\right)$ are neglected and the effective flux becomes simply $F$ (a valid assumption at low coverages), the rate equations can be solved analytically. However, the solution is expressed in terms of intractable nested integral expressions, and is of little use to us. In the case we study here, in which the rejection terms are taken into account, no such solution exists and the equations are integrated numerically.

\section{Analysis and Results}

\subsection{Methods}

We will now examine to what extent the rate equation model can describe the experimental results. To this end we performed numerical integration of Eqs. (3a)-(3b) with the aid of a Bulirsch-Stoer stepper algorithm (Press et al. 1992). The result of the integration is a set of TPD curves that are a function of the chosen set of parameters. A standard TPD experimental run includes the time dependence of the flux $F(t)$ and temperature $T(t)$ as well as the four parameters $E_{0}, E_{1}, E_{2}$ and $\mu$. The temperature $T(t)$ is measured directly via a thermocouple. The flux $F(t)\left(s^{-1}\right)$ is estimated as described elsewhere (Vidali et al. 1998B). An approximate value for $F(t)$, in the required units of $M L s^{-1}$, can be obtained by integrating the TPD spectra, generating the total yield of the various experiments. The flux is then obtained from the exponential fit indicated by Langmuir kinetics. It is important to stress that this is a lower bound value for the flux, and this value is reached only if there is no $\mathrm{H}$ desorption at all. We are now left with the four parameters $E_{0}, E_{1}, E_{2}$ and $\mu$ which are assumed to be independent of the flux or temperature. These parameters form a four dimensional space that has to be explored in order to find the values for which the calculated TPD curves provide the best fit to the experimental TPD ones.

The merit function to be minimized in the fitting procedure is the standard $\chi^{2}$ function, which is the sum over the squares of the differences between the experimental points and the calculated ones. Another possibility that we considered was to compare the derivatives of the experimental TPD curves with the ones of the simulation (again using $\chi^{2}$ ). This 
possibility required using the Savitzki-Golay filtering (Press et al. 1992) in order to obtain a reasonably smooth derivative from the experimental data, because of the well known increase in the noise to signal ratio in any derivative of measured data.

To obtain the parameters that best fit the experimental data one needs to probe the parameter space and find the set of values of the parameters that give rise to the global minimum of the merit function. The probing procedure we used is based on random search. A point in the four dimensional parameter space is randomly picked. A numerical integration of the model's equations is performed with the chosen parameters. The merit function obtained from the comparison of the resulting curve with the experimental curve is then evaluated. If this set represents an improvement over the current minimum, the new point is accepted as the new minimum. If, however, the new set did not score lower (i.e. better) than the known minimum, it was promptly discarded. The probability distribution for picking the next random set of parameters was taken to be a Lorentzian centered around the current set of parameters and thus favored nearby points. The relatively slow drop-off of the Lorentzian function also allowed the occasional taking of longer steps and thus prevented the process from getting stuck in a local minimum.

\subsection{Results}

The experimental TPD curves and the fits obtained by the rate equations are shown in Figs. 1 and 2 for the olivine sample and in Figs. 3 and 4 for the amorphous carbon sample. The parameters obtained in the fitting procedure for the two samples are shown in Table 1. The parameter set generated for each sample represents a simultaneous best fit for all the six TPD curves. Fitting each curve separately typically produce better fits, but at the expense of an increased range in the values of the parameters. These variations allow us to generate approximate error estimates. It is found that the energy barriers $E_{0}$ and $E_{2}$ are very well determined by this process (to within several tenths of a meV). The barrier $E_{1}$ is not as well determined, and its values given above, for both samples, are to be taken as lower bounds to the correct value (within $3 \mathrm{meV}$ ). The parameter $\mu$ is determined to within \pm 0.1 , and thus justifies our assumption, within this model, that not all $\mathrm{H}_{2}$ molecules immediately desorb upon recombination. Attempting to artificially force $\mu=0$ and do the fits with the remaining three parameters, degrades the fit substantially and cannot recreate the entire range of behavior of the data simultaneously.

Although Eq. (2) can be used to fit the entire range of experimental TPD results, this equation, which includes a single activation energy, does not provide as much insight 
as our model [Eqs. (3)]. For example, applying Eq. (2) in the case of Fig. 1 (olivine, 1st order desorption kinetics), we use $\beta=1$ and arrive at $E_{d}=26.8 \mathrm{meV}$. This is equivalent to the $E_{2}$ found for olivine using this model. However, as we shift to Fig. 2 (olivine, 2nd order desorption kinetics), we must now take $\beta=2$, which results in $E_{d}=24 \mathrm{meV}$. Here the rate limiting process is the diffusion on the surface, and therefore $E_{d}$ is closer to $E_{0}$. Unlike Eq. (2), our model does not require setting a parameter (such as $\beta$ ). Furthermore, it provides the best-fit values of each of the relevant activation energies for both the first and second order kinetics and using the same framework.

The model [Eqs. (3)] can also describe the steady state conditions which are reached when both the flux and the temperature are fixed. The steady state solution is then easily obtained by setting $\dot{N}_{1}$ and $\dot{N}_{2}$ to 0 and solving the quadratic equation for $N_{1}$. The coefficients $P_{1}, P_{2}$ and $\alpha$ in the rate equations are temperature dependent and under the steady state assumption maintain constant values. The complete solution under these assumptions takes the form (after neglecting the unphysical possibility of the negative root):

$$
\begin{aligned}
& N_{1}=\frac{\sqrt{\left(P_{1}+F\right)^{2}+8\left(\alpha F+\frac{\mu \alpha F^{2}}{2 P_{2}}\right)}-\left(P_{1}+F\right)}{4\left(\alpha F+\frac{\mu \alpha F^{2}}{2 P_{2}}\right)} \\
& N_{2}=\frac{\left(P_{1}+F\right)^{2}+4\left(\alpha F+\frac{\mu \alpha F^{2}}{2 P_{2}}\right)-\left(P_{1}+F\right) \sqrt{\left(P_{1}+F\right)^{2}+8\left(\alpha F+\frac{\mu \alpha F^{2}}{2 P_{2}}\right)}}{8\left(\alpha+\frac{\mu \alpha F}{2 P_{2}}\right)^{2}} .
\end{aligned}
$$

Note that when the rejection terms in the flux are neglected, the expressions for the steady state coverages and recombination rate become significantly simpler (Biham et al. 1998). These solutions may be useful in the study of recombination processes in the interstellar medium where steady state conditions may be relevant.

In this work, we present a model that captures the kinetics of the diffusionrecombination-desorption process. The parameters thus obtained can then be used to study the astrophysically relevant cases. For example, by assuming steady state conditions, we can obtain the recombination efficiency as a function of flux $F$ and temperature $T$ for a range of parameters that goes from the astrophysically relevant (extremely low flux, $10-15 \mathrm{~K}$ ) to the ones used in the laboratory (low flux, $5-30 \mathrm{~K}$ ). The recombination efficiency is defined as the ratio between the production rate $R$ [Eq. (7)] and the deposition rate $F / 2$ (in molecules).

Varying $T$ and $F$ over the astrophysically relevant range we can identify the regions in which there is non-negligible recombination efficiency. The recombination efficiency as a function of $T$ and $F$ is shown in Fig. 5 for olivine and in Fig. 6 for amorphous carbon. 
The main conclusion from these figures is that the recombination efficiency is highly temperature dependent. There is an efficiency window along the temperature axis which shifts to higher temperatures as the flux is increased. It is found that under astrophysically relevant irradiation rates the efficiency for olivine drops off at approximately $7 \sim 8 \mathrm{~K}$, while for amorphous carbon it drops off only at $13 \sim 14 \mathrm{~K}$.

We find that, for both samples, the parameter $\mu$ (the probability of an $\mathrm{H}_{2}$ molecule to remain on the surface upon recombination) has little effect on the production rate $R$ of molecular hydrogen under steady state conditions. This is easy to understand, since under steady state conditions the production rate $R$ must be equal to the recombination rate on the surface, and, thus, must be independent of $\mu$. The coverage of hydrogen molecules on

the surface is adjusted accordingly. Similarly, the energy barrier for desorption of molecular hydrogen, $E_{2}$, has little effect on the recombination efficiency, under steady state conditions, as long as it remains significantly smaller than the barrier for atomic desorption, $E_{1}$.

\section{Discussion}

Amorphous materials in general, and their surfaces in particular, are difficult to characterize, due to their irregular structure and composition. In the experiments analyzed here, it gives rise to some uncertainty about the role of quantum effects. In principle, tunneling of $\mathrm{H}$ atoms should be considered. However, the experiments indicate that quantum effects appear to be small, as the mobility of the hydrogen atoms is very low at the lower irradiation temperatures (Pirronello et al. 1997b). Another issue is that in the experiments the desorption rates of HD were measured and the extrapolation to processes involving $\mathrm{H}$ and $\mathrm{H}_{2}$ is non-trivial. This difficulty is unavoidable, however, due to the large background noise in the measurements of $\mathrm{H}_{2}$ production. Careful calibration of the apparatus was used in generating the necessary extrapolation coefficients. Taking isotopic effects into account in the rate equations is not easy for this type of surfaces, since the shape of the energy surface is not known. An attempt was made to introduce separate fitting parameters for $\mathrm{H}$ and $\mathrm{D}$, while keeping $\mu=0$ (to maintain the same number of parameters). This fit was considerably worse than the ones presented here. Since isotopic effects enter as a shift in the activation energies of the various processes described here, it is fair to consider an "effective" hydrogen atom in the simulations.

Despite these obstacles, we obtained good fits to the experimental TPD curves using numerical integration of the rate equation model. This seems to indicate that most of the processes that occur at the microscopic level are captured by the dynamics of the rate 
equations, or at least they average out to this global dynamics.

According to common physical intuition based on long range forces and the values of atomic and molecular polarizabilities, $\mathrm{H}_{2}$ is expected to be bound to the substrate more tightly than H. This, however, does not appear to be the case: the parameters calculated numerically, for both samples, put the $\mathrm{H}$ atoms in deeper wells than $\mathrm{H}_{2}$. This feature must signal the fact that more complicated interactions (based also on shorter range forces) are taking place.

The recombination efficiency diagrams (Figs. 5-7) include two regions of low efficiency on both sides of the high efficiency window. The asymptotic steady state coverage in the region on the left hand side approaches unity, while everywhere else is very small. This is due to the fact that at such low temperatures atoms remain stuck in place and barely diffuse; thus, recombination is inhibited. These atoms accumulate on the surface blocking adsorption sites ("Langmuir rejection"). However, this high coverage regime is not one that we can extrapolate to with any certainty within this rate equation model. As already suggested in Pirronello et al. (1999), mechanisms such as Eley-Rideal or diffusion by tunneling, which are not taken into account in the model, may become significant in this regime. Nevertheless, we can speculate that if the Langmuir rejection remains significant even at higher coverages, the trend in recombination efficiency shown should remain qualitatively correct. Luckily, such low temperatures are rarely of astrophysical interest. For olivine, at temperatures above $6.5 \mathrm{~K}$, the calculated asymptotic coverage is very low and well within the regimes of experimentation and subsequent numerical simulation; consequently, the relevance of the model is justified.

We find that the recombination efficiency on olivine is high in the temperature range of roughly $5-10 \mathrm{~K}$; however, this temperature range is lower than the one encountered in the interstellar clouds. Therefore, we believe that this material is not a very likely candidate for an efficient catalyst of hydrogen recombination in interstellar space.

The recombination efficiency on amorphous carbon, on the other hand, behaves differently (recall Fig. 6). Here we see that the final drop in efficiency of $\mathrm{H}_{2}$ recombination is at higher temperatures, and amorphous carbon seems to be a more appropriate candidate of interstellar grains on which hydrogen may recombine with high efficiency.

In a previous paper (Biham et al. 1998) two limiting expressions were obtained for the $\mathrm{H}_{2}$ production rate per unit volume under steady state conditions. Such expressions are linear (when $\alpha F \gg P_{1}^{2}$ ) or quadratic (when $\alpha F \ll P_{1}^{2}$ ) in the flux of gas phase atoms. Alternatively, they are independent (when $\alpha F \gg P_{1}^{2}$ ) or quadratically dependent (when $\left.\alpha F \ll P_{1}^{2}\right)$ on the coverage of $\mathrm{H}$ adatoms. Note that in Biham et al. (1998) the value $\mu=1$ 
was taken. In that work, we studied the steady state behavior of the rate equations for the processes described here, where the value of $\mu$ does not affect the recombination efficiency. The first of the two limits of the steady state production rate of molecular hydrogen per unit volume coincides with the expression of Hollenbach's et al. [see Eq. (四)]. However, it is found that the second of the two limits, which is valid when there is a very low coverage of $\mathrm{H}$ adatoms on interstellar grains, coincides with the expression proposed by Pirronello et al. (1997b):

$$
R_{\mathrm{H}_{2}}=\left(n_{\mathrm{H}} v_{\mathrm{H}} \sigma \xi t_{\mathrm{H}}\right)^{2} n_{\mathrm{g}} \alpha \gamma^{\prime},
$$

where $t_{\mathrm{H}}=1 / P_{1}$ and $\gamma^{\prime}$ is the probability that two $\mathrm{H}$ adatoms recombine upon encountering (taking into account that there might be an activation energy for recombination).

In Fig. 7 we present a contour diagram of the recombination efficiency on the carbon surface. On this diagram, the parameter values for which $\alpha F=P_{1}^{2}$ are plotted as a starred line. In the region on the left hand side of the starred line, the calculated $R_{H_{2}}$ approaches the value obtained when $\alpha F \gg P_{1}^{2}$. On the right hand side of the starred line, the calculated $R_{H_{2}}$ approaches the value obtained in the limit of $\alpha F \ll P_{1}^{2}$. The transition from the regime where $\alpha F \gg P_{1}^{2}$ holds to the other, $\alpha F \ll P_{1}^{2}$, is quite rapid because of the exponential nature of the temperature dependence. We conclude that the two cases discussed in (Biham et al. 1998) apply in a wide region of the diagrams (Fig.5-7).

The diagrams described above apply under conditions close to steady state. This is not necessarily the case in the interstellar clouds. If the entire cloud is far from steady state, one must return to the original rate equation model and take into account the time dependence of the flux and temperature.

The values obtained for $\mu$ imply a non-negligible probability for the hydrogen molecules to remain on the surface instead of being immediately ejected into the gas phase. This result was found to be unavoidable within the assumption of the model used here. There are various mechanisms for efficient heat transfer from the molecule to the surface. These may dissipate the excess energy and prevent immediate desorption. One possible mechanism may be due to the very irregular structure of the sample surfaces: olivine was mechanically polished (hence there should be grooves of hundreds of Angstroms in width and depth). Atomic force microscopy (AFM) images of the olivine surface show such a rugged landscape at submicron scale, while amorphous carbon is composed of grains of the size of a hundred Angstrom. A definite possibility (more relevant in the amorphous carbon case, as the somewhat larger $\mu$ value suggests) is that hydrogen molecules, even if promptly released upon formation, do not necessarily go directly into the vacuum but undergo a multiple series of collisions in which part of their energy is released to the solid with a 
subsequent re-adsorption. Such mechanism was identified for $\mathrm{H}$ and D atoms impinging on an amorphous ice particle (Buch \& Zhang 1991). More experiments are needed in order to elucidate the nature of recombination process at the atomic scale and to obtain directly a value for $\mu$.

\section{Summary}

Experimental results on the formation of molecular hydrogen on various materials and conditions relevant to interstellar clouds were analyzed using rate equations. By fitting the results of a TPD experiment to a rate equation model, four essential parameters of the process were obtained. These are the activation energy barriers for atomic hydrogen diffusion and desorption, the barrier for molecular hydrogen desorption, and the probability of spontaneous desorption of a hydrogen molecule upon recombination. The results compare favorably with what is obtained from the Polanyi-Wigner equation. Furthermore, the model represents a generalization that allows us to describe both first and second order processes (or even a combination of the two) within a single model.

In this work we have shown a procedure to extrapolate data taken under conditions available in the laboratory to values that should hold in astrophysical environments, and to determine the efficiency of various surfaces as catalysts in molecule production. Polycrystalline olivine was shown to be inefficient as a catalyst in the relevant temperature/flux regime. On amorphous carbon a higher efficiency was reached due to higher desorption/diffusion barriers that cause a rise in the recombination efficiency at the relevant temperatures.

The proposed model and methods of analysis are neither complete nor definitive, but nonetheless represent a serious improvement over what has been used so far to predict the behavior of hydrogen on astrophysically relevant surfaces.

\section{Acknowledgments}

G.V. acknowledges support from NASA grant NAG5-6822. 


\section{REFERENCES}

Aronowitz S., \& Chang S., 1985, ApJ, 293, 243.

Biham, O., Furman, I., Katz, N., Pirronello, V., \& Vidali, G. 1998, MNRAS, 296, 869.

Buch, V., \& Zhang, Q. 1991, Astrophys. J. 379, 647.

Chan, C.M., Aris, R., \& Weinberg, W.H. 1978, Appl. Surf. Sci. 1, 360.

Duley, W.W., \& Williams, D.A. 1984, Interstellar Chemistry (Academic Press, London).

Duley, W.W., \& Williams, D.A. 1986, MNRAS, 223, 177.

Farebrother, A., Fisher, A., and Clary, D.C., 1999, in preparation.

Gould, R.J. and Salpeter, E.E. 1963 Astrophys. J. 138, 393.

Hollenbach, D., \& Salpeter, E.E. 1970, J. Chem. Phys. 53, 79.

Hollenbach, D., \& Salpeter, E.E. 1971, ApJ, 163, 155.

Hollenbach, D., Werner M.W., \& Salpeter, E.E. 1971, ApJ, 163, 165.

Lin, J., \& Vidali, G. 1996, in "The Cosmic Dust Connection", Ed. by M. Greenberg, (Kluwer Academic Press) p. 323.

Masuda, K., Takahashi, J., and Mukai, T., 1998, A\&A, 330, 773.

Pirronello, V. \& Averna D. 1988, A\&A, 201, 196

Pirronello, V., Liu, C., Shen L., \& Vidali, G. 1997a, ApJ, 475, L69.

Pirronello, V., Biham, O., Liu, C., Shen L., \& Vidali, G. 1997b, ApJ, 483, L131.

Pirronello, V., Liu, C., Roser J.E, \& Vidali, G. 1999, A\&A, in press.

Press, W.H., Teukolsky S.A., Vetterling, W.T, \& Flannery B.P. 1992, "Numerical Recipes in C, The Art of Scientific Computing" (Cambridge University Press).

Rettner, C.T., \& Auerbach, D.J., 1996, Surf. Sci. 357, 602.

Sandford S.A., \& Allamandolla L.J. 1993, ApJ, 409, L65.

Scoles, G., \& Herman, L.J.F.

Smoluchowski, R. 1981, Astrophys. Space Sci. 75, 353.

Smoluchowski, R. 1983, J. Phys. Chem. 87, 4229.

Takahashi, J, Masuda, K., and Nagaoka, M., 1999, A\&A, in press.

Vidali G., Pirronello V., Liu, C., Shen L.Y., 1998a, Astrophys. Lett. Comm., 35, 423.

Vidali., G., Roser, J. Liu, C., Pirronello, V., Biham, O., 1998b, Proceedings of the NASA Laboratory Space Science Workshop, (Cambridge, Mass. April 1-3 1998), in press. 
Williams D.A. 1998, Faraday Discussion N.109, p.1.

Williams D.A. 1968, ApJ, 151, 935.

Winkler, A. 1998, Appl. Phys. A., 67, 637.

Table 1: The four parameters obtained by the fitting of the TPD curves for polycrystalline olivine and amorphous carbon. $E_{0}$ is the barrier for atomic diffusion, $E_{1}$ and $E_{2}$ are the barriers for atomic and molecular desorption respectively, $1-\mu$ is the probability of spontaneous desorption of a newly formed $\mathrm{H}_{2}$ molecule.

\begin{tabular}{||c|c|c|c|c||}
\hline material & $E_{0}(\mathrm{meV})$ & $E_{1}(\mathrm{meV})$ & $E_{2}(\mathrm{meV})$ & $\mu$ \\
\hline \hline olivine & 24.7 & 32.1 & 27.1 & 0.33 \\
\hline amorphous carbon & 44.0 & 56.7 & 46.7 & 0.413 \\
\hline
\end{tabular}




\section{Figure Captions}

Fig. 1. - TPD curves for higher coverage experiments on an olivine slab. Irradiation times are (in minutes) $8.0(\bullet), 5.5(\times)$ and $2.0(+)$. Fits are in solid lines.

Fig. 2.- TPD curves for lower coverage experiments on an olivine slab. Irradiation times are (in minutes) $0.55(\bullet), 0.2(\times)$ and $0.07(+)$. Fits are in solid lines.

Fig. 3. - TPD curves for higher coverage experiments on amorphous carbon. Irradiation times are (in minutes) $32.0(\bullet), 16.0(\times)$ and $8.0(+)$. Fits are in solid lines.

Fig. 4.- TPD curves for lower coverage experiments on amorphous carbon. Irradiation times are (in minutes) $4.0(\bullet), 2.0(\times)$ and $1.0(+)$. Fits are in solid lines.

Fig. 5.- Recombination efficiency at steady state of the olivine slab as a function of $\log _{10}(F)$ (flux in $M L / s e c$ ) and $\mathrm{T}$ (temperature in $K$ ).

Fig. 6.- Recombination efficiency at steady state of amorphous carbon as a function of $\log _{10}(F)$ (flux in $\left.M L / s e c\right)$ and $\mathrm{T}$ (temperature in $\left.K\right)$.

Fig. 7.- Contour plot of the recombination efficiency at the steady state for amorphous carbon, as a function of $\log _{10}(F)$ (flux in $M L / s e c$ ) and $\mathrm{T}$ (temperature in $K$ ). The starred line represents $\alpha F=P_{1}^{2}$. 


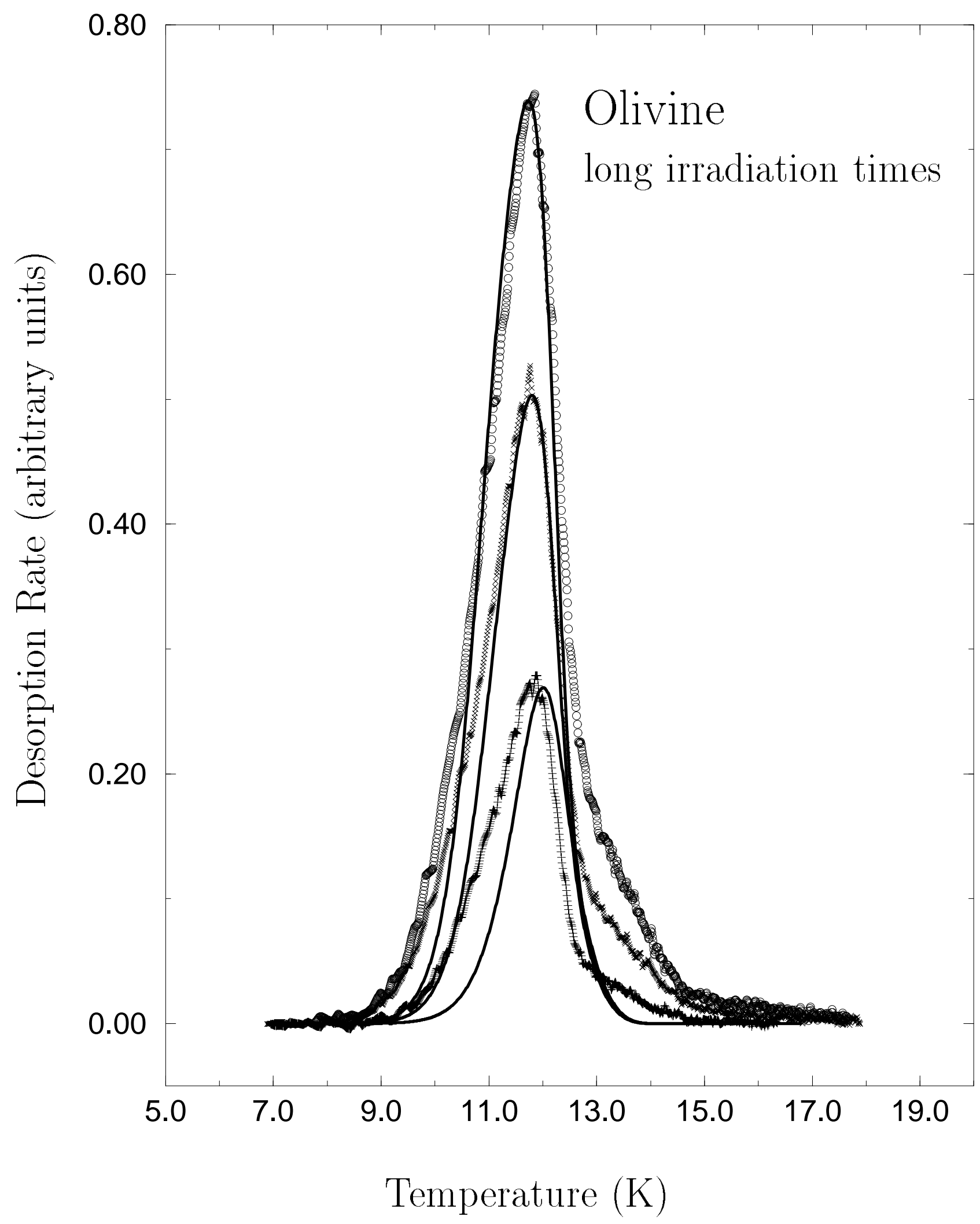

FIG. 1 


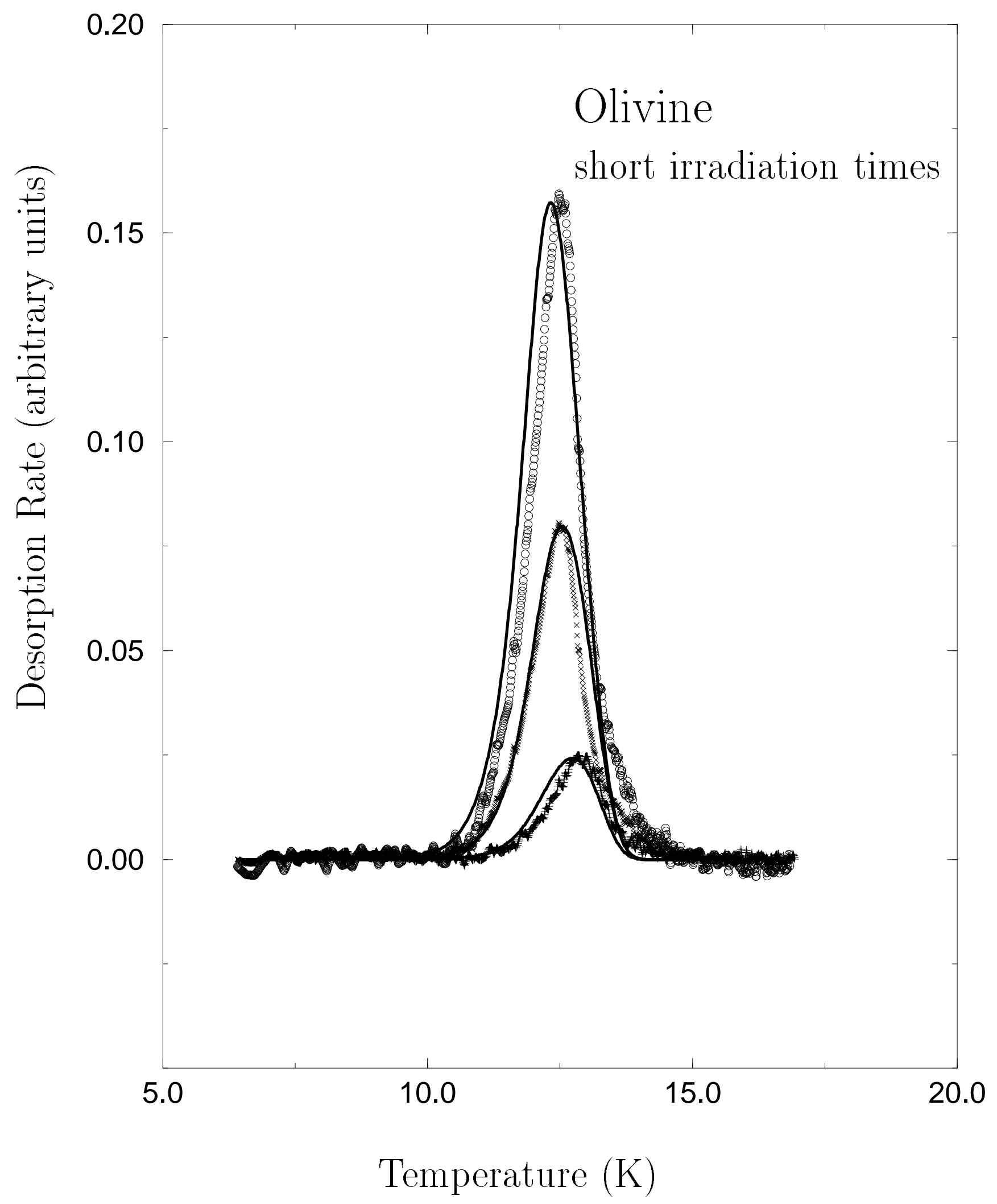

FIG. 2 
This figure "fig3.gif" is available in "gif" format from: http://arxiv.org/ps/astro-ph/9906071v1 
This figure "fig4.gif" is available in "gif" format from: http://arxiv.org/ps/astro-ph/9906071v1 
This figure "fig5.gif" is available in "gif" format from: http://arxiv.org/ps/astro-ph/9906071v1 
This figure "fig6.gif" is available in "gif" format from: http://arxiv.org/ps/astro-ph/9906071v1 


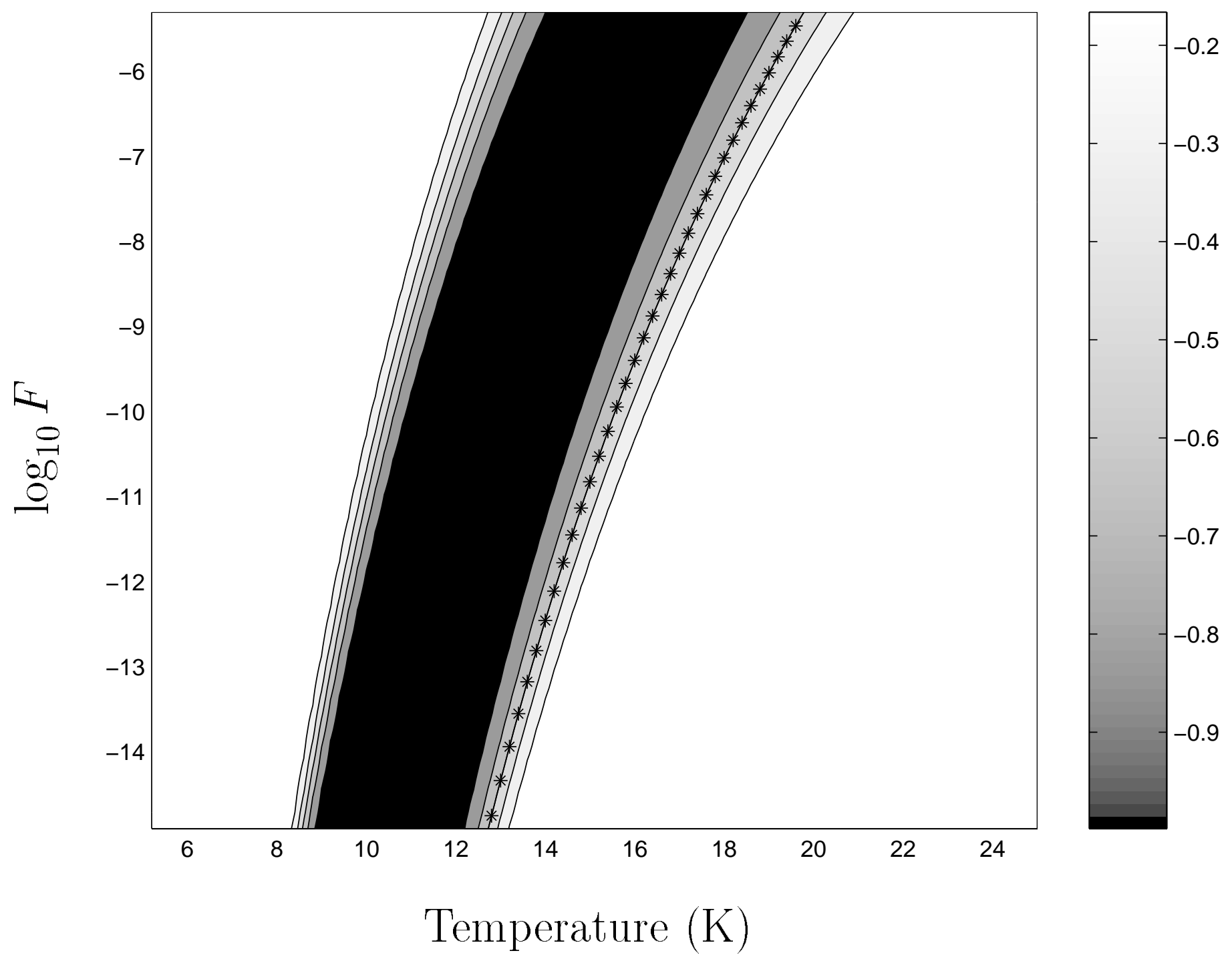

FIG. 7 